\title{
Effect of Multiple Doses of Omeprazole on the Pharmacokinetics, Safety, and Tolerability of Roxadustat in Healthy Subjects
}

\author{
Dorien Groenendaal-van de Meent ${ }^{1} \cdot$ Martin den Adel $^{1} \cdot$ Jan van Dijk $^{1} \cdot$ Begona Barroso-Fernandez $^{2}$. \\ Rachid El Galta ${ }^{3}$ Georg Golor ${ }^{4} \cdot$ Marloes Schaddelee $^{5}$
}

Published online: 11 May 2018

(c) The Author(s) 2018

\begin{abstract}
Background and Objectives Roxadustat is an orally active hypoxia-inducible factor prolyl hydroxylase inhibitor for the treatment of anemia in chronic kidney disease. This study investigated the effect of multiple daily oral doses of omeprazole on the pharmacokinetics, safety, and tolerability of a single oral dose of roxadustat.

Methods This phase 1, open-label, two-period, one-sequence, crossover study enrolled healthy subjects. During Period 1, subjects received a single oral dose of $100 \mathrm{mg}$ roxadustat. After a $\geq 7$-day washout, subjects started Period 2 and received daily oral doses of $40 \mathrm{mg}$ omeprazole on Days $1-9$, and a single oral dose of $100 \mathrm{mg}$ roxadustat on Day 7. Roxadustat pharmacokinetics were assessed on Days $1-4$ in Period 1 and on Days 7-10 in Period 2. Primary endpoints were area under the concentration-time
\end{abstract}

Electronic supplementary material The online version of this article (https://doi.org/10.1007/s13318-018-0480-z) contains supplementary material, which is available to authorized users.

Dorien Groenendaal-van de Meent

Dorien.Groenendaal@astellas.com

Martin den Adel

Martin.denAdel@astellas.com

Jan van Dijk

Jan.vanDijk@astellas.com

Begona Barroso-Fernandez

Begona.Barroso@astellas.com

Rachid El Galta

Rachid.ElGalta@astellas.com

Georg Golor

g.golor@gmail.com

Marloes Schaddelee

Marloes.Schaddelee@astellas.com profile from the time of dosing extrapolated to infinity $\left(\mathrm{AUC}_{\mathrm{inf}}\right)$ and maximum concentration $\left(\mathrm{C}_{\max }\right)$. Safety was assessed by vital signs, laboratory tests, electrocardiograms, and nature, frequency, and severity of treatmentemergent adverse events (TEAEs).

Results Eighteen subjects were enrolled. The geometric least squares mean ratio for both $\mathrm{AUC}_{\mathrm{inf}}$ and $\mathrm{C}_{\max }$ of roxadustat (with omeprazole/alone) was 104.5\%; $90 \%$ confidence intervals were within the no-effect boundaries of 80.0 and $125.0 \%$, indicating no significant effect of omeprazole on the pharmacokinetics of roxadustat. No serious TEAEs were reported.

Conclusion Multiple daily oral doses of $40 \mathrm{mg}$ omeprazole had no significant effect on the pharmacokinetics of a single oral dose of $100 \mathrm{mg}$ roxadustat. Roxadustat was considered safe and well tolerated when administered alone or in combination with multiple daily oral doses of $40 \mathrm{mg}$ omeprazole in healthy subjects.

1 Clinical Pharmacology and Exploratory Development, Astellas Pharma Europe B.V., Sylviusweg 62, 2333 BE Leiden, The Netherlands

2 Drug Discovery Research/Bioanalysis Europe, Astellas Pharma Europe B.V., Sylviusweg 62, 2333 BE Leiden, The Netherlands

3 Data Science Biostatistics, Astellas Pharma Europe B.V., Sylviusweg 62, 2333 BE Leiden, The Netherlands

4 PAREXEL, Spandauer Damm 130, 14050 Berlin, Germany

5 Business Development, Transaction Execution Group, Astellas Pharma Europe B.V., Sylviusweg 62, 2333 BE Leiden, The Netherlands 


\section{Key Points}

Administration of multiple daily oral doses of $40 \mathrm{mg}$ omeprazole had no significant effect on the pharmacokinetics of a single oral dose of $100 \mathrm{mg}$ roxadustat.

The coadministration of multiple daily oral doses of $40 \mathrm{mg}$ omeprazole and a single oral dose of $100 \mathrm{mg}$ roxadustat was considered safe and well tolerated in healthy subjects.

\section{Introduction}

Anemia is a common complication in patients with chronic kidney disease (CKD) that is caused, in part, by the inability of the failing kidneys to maintain adequate synthesis of erythropoietin (EPO), resulting in reduced levels of hemoglobin. Other etiological factors of anemia in CKD include altered iron homeostasis, shortened erythrocyte survival, and inflammation [1]. Prevalence of anemia increases with the severity of CKD [2,3] and is associated with an increased risk of cardiovascular comorbidities [4], prolonged hospital stay [5], reduced quality of life, and activity impairment [6].

Current concerns regarding the safety of erythropoiesisstimulating agent (ESA) therapy have caused a reduction in ESA use, leading to an unmet need for treatment of anemia in CKD [7, 8]. A novel class of therapeutic agents for the treatment of anemia in patients with CKD targets hypoxiainducible factor, a family of oxygen-sensitive transcriptional factors that are modulated by prolyl hydroxylase enzymes and play a key role in regulating erythropoiesis [7]. Hypoxia-inducible factor prolyl hydroxylase inhibitors (HIF-PHIs) increase endogenous EPO to levels that are observed during an acute bleed or chronic hypoxia states, which are significantly lower than the supraphysiologic peaks observed during treatment with intravenous ESA. Therefore, HIF-PHIs may provide an effective treatment for anemia in CKD without the adverse outcomes associated with ESA therapy $[9,10]$.

Roxadustat (ASP1517, FG-4592, AZD9941) is an orally active HIF-PHI currently in phase 3 investigation for the treatment of anemia in patients with CKD who are on or not on dialysis. Roxadustat has previously demonstrated safety and efficacy in phase 2 trials in patients with dialysis-dependent [11-13] and non-dialysis-dependent [9, 12, 14] CKD. In addition to transiently increasing endogenous EPO levels, roxadustat has been shown to reduce the dysregulation of iron metabolism associated with CKD by increasing serum transferrin, intestinal iron absorption, and release of stored iron, in a dose-dependent manner [9, 12]. Roxadustat reaches its maximum plasma concentration in approximately $2 \mathrm{~h}$ after oral administration and has a terminal elimination half-life $\left(\mathrm{t}_{1 / 2}\right)$ of $12-14 \mathrm{~h}$ after a single dose [15]. Roxadustat is predominantly eliminated by phase I oxidation (cytochrome P450 [CYP] $2 \mathrm{C} 8$ ) and phase II conjugation (glucuronidation via uridine diphosphate-glucuronosyltransferase [UGT 1A9] and glucosidation). Furthermore, roxadustat is highly bound to proteins in human plasma, predominantly albumin. A DDI study in healthy volunteers revealed that roxadustat does not impact the maximum concentration $\left(\mathrm{C}_{\max }\right)$ or area under the concentration-time profile from the time of dosing extrapolated to infinity ( $\mathrm{AUC}_{\mathrm{inf}}$ ) of S- or R-warfarin, but does delay the time to reach maximum concentration $\left(\mathrm{t}_{\max }\right)$ of $\mathrm{R}$-warfarin by approximately $1 \mathrm{~h} \mathrm{[16].} \mathrm{The}$ solubility of roxadustat in buffered aqueous solutions is $\mathrm{pH}$ dependent and increases from $0.001 \mathrm{mg} / \mathrm{mL}$ in a simulated gastric fluid buffer at $\mathrm{pH} 1.2$ to $3.7 \mathrm{mg} / \mathrm{mL}$ in a $50 \mathrm{mM}$ potassium phosphate buffer at $\mathrm{pH} 7.5$ (data on file). Omeprazole is a proton pump inhibitor commonly used for the treatment of gastroesophageal reflux disease and peptic ulcer disease [17]. Omeprazole increases gastric $\mathrm{pH}$ and may therefore increase the solubility of roxadustat and affect its absorption.

To evaluate whether gastric $\mathrm{pH}$-raising drugs such as proton pump inhibitors impact roxadustat absorption, this study evaluated the impact of multiple daily oral doses of omeprazole on the pharmacokinetics of a single oral dose of roxadustat, as well as the safety and tolerability of a single oral dose of roxadustat alone or in combination with multiple daily oral doses of omeprazole.

\section{Methods}

\subsection{Study Design}

This was a phase 1 , open-label, one-sequence, crossover study (1517-CL-0527; EudraCT 2014-000666-22) conducted at a single center (PAREXEL Early Phase Clinical Unit) in Berlin, Germany, consisting of a screening period (Day -22 to Day -2 ), followed by two treatment periods separated by a $\geq 7$-day washout phase. During Period 1, subjects were admitted to the clinic on Day -1 and received a single oral dose of $100 \mathrm{mg}$ roxadustat (ASP1517; Astellas Pharma, Inc.) on Day 1. Subjects remained in the clinic and were discharged on Day 4. Blood samples for roxadustat pharmacokinetic assessments were collected on Days 1-4. The starting doses in current phase 3 studies of patients with 
CKD not on dialysis range from 70 to $100 \mathrm{mg}$ three times weekly and can be up- or down-titrated over a range of 20-300 mg three times a week. Therefore, the selected dose of $100 \mathrm{mg}$ roxadustat truly represents a medium range and widely used therapeutic dose. This dose level in the present DDI study allowed for potential increases in roxadustat exposure due to the presence of omeprazole without any safety concerns [9]. After a $\geq 7$-day washout period, subjects were re-admitted to the clinic on Day -1 of Period 2. During Period 2, subjects received daily oral doses of $40 \mathrm{mg}$ omeprazole (Antra MUPS ${ }^{\circledR}$, AstraZeneca) on Days 1-9 and a single oral dose of $100 \mathrm{mg}$ roxadustat on Day 7. Blood samples for roxadustat pharmacokinetic assessments were collected on Days 7-10 and blood samples for omeprazole pharmacokinetic assessments were collected on Days 1, 3, 5, and 7-10. Subjects were discharged on Day 10, and returned to the clinic for an end of study visit 5-9 days later for safety assessments. Since the recommended dose of omeprazole for the treatment of gastroesophageal reflux disease and peptic ulcer disease is $20-40 \mathrm{mg}$ daily [18], a regimen of daily administration of high doses of omeprazole (40 mg) on Days 1-7, before administration of a single oral dose of $100 \mathrm{mg}$ roxadustat on Day 7, was chosen to maximize the interaction potential and to closely mimic real-life treatment conditions. Blood samples for pharmacokinetic analyses of roxadustat were collected pre- and post-dose at prefixed intervals during Period 1 and Period 2.

This study was conducted in accordance with the clinical study protocol, Good Clinical Practice, the International Council on Harmonisation guidelines, applicable regulations and guidelines governing clinical study conduct, and the ethical principles of the Declaration of Helsinki. The protocol was approved by an Independent Ethics Committee (Landesamt für Gesundheit und Sociales [Ethik-Kommission des Landes Berlin]) and all subjects provided written informed consent.

\subsection{Study Objectives}

The primary objective was to determine the effect of multiple daily oral doses of $40 \mathrm{mg}$ omeprazole on the pharmacokinetics of a single oral dose of $100 \mathrm{mg}$ roxadustat. The secondary objective was to evaluate the safety and tolerability of a single oral dose of $100 \mathrm{mg}$ roxadustat alone and in combination with multiple daily oral doses of $40 \mathrm{mg}$ omeprazole.

\subsection{Study Population}

Healthy male or female subjects, aged 18-55 years, with a body mass index of $18.5-29.9 \mathrm{~kg} / \mathrm{m}^{2}$ were enrolled in this study. Female subjects were either of non-childbearing potential or must have had a negative serum pregnancy test at Day -1 and had to agree to use two forms of highly effective birth control from the time of screening to 28 days after final dosing. Male subjects, and their female partners of childbearing potential, had to agree to use two highly effective forms of birth control, and male subjects had to agree to not donate sperm from screening to 28 days after final dosing.

Key exclusion criteria included pregnancy within 6 months and breastfeeding within 3 months before screening; liver function tests above $1.5 \times$ the upper limit of normal; use of any prescription or nonprescription drugs (including oral contraceptives) other than occasional paracetamol (up to $2 \mathrm{~g}$ /day) during the 2 weeks prior to the administration of the study drug until the end of study visit; a history of drinking $>21$ units (male subjects) or $>14$ units (female subjects) of alcohol (1 unit $=10 \mathrm{~g}$ pure alcohol, $250 \mathrm{~mL}$ of beer [5\%], $35 \mathrm{~mL}$ of spirits [35\%], or $100 \mathrm{~mL}$ of wine [12\%]) per week or drugs of abuse in the 3 months before admission; and use of any drugs that induced metabolism within 3 months prior to admission. A complete list of inclusion and exclusion criteria is available in the Online Resource.

\subsection{Study Drug Administration}

On Day 1 of Period 1, roxadustat was provided as a single $100 \mathrm{mg}$ tablet with $240 \mathrm{~mL}$ of water after an overnight fast of at least $8 \mathrm{~h}$, with the first meal provided $4 \mathrm{~h}$ after dosing. On Days 1-6 and Days 8 and 9 of Period 2, omeprazole was provided as a dose of $2 \times 20 \mathrm{mg}$ tablets once daily (for a total daily dose of $40 \mathrm{mg}$ ) with $240 \mathrm{~mL}$ water after an overnight fast of at least $8 \mathrm{~h}$, and $30 \mathrm{~min}$ before breakfast. On Day 7 of Period 2, omeprazole was dosed first and roxadustat was dosed $2 \mathrm{~h}$ later; the first meal on Day 7 was provided $4 \mathrm{~h}$ after roxadustat administration.

\subsection{Study Assessments}

\subsubsection{Pharmacokinetic Assessments}

Blood sampling for roxadustat was conducted at predose, 30 min, 1, 2, 3, 4, 5, 6, 8, 12, 16, 24, 36, 48, 60, and $72 \mathrm{~h}$ postdose on Days 1-4 of Period 1 and on Days 7-10 of Period 2. Blood sampling for omeprazole was conducted at predose on Days 1, 3, 5, and 7, and 30 min, 1, 2, 3, 4, 6, and $8 \mathrm{~h}$ postdose on Days 7 and predose on Days 8 and 9 . Blood samples were obtained via a peripherally placed intravenous cannula or via direct venipuncture and collected into 3-mL tubes of sodium heparin. Blood samples were shipped to Astellas Drug Discovery Research/Bioanalysis Europe, and concentrations of roxadustat in human Na-heparin plasma were measured using a liquid chromatography tandem mass spectrometry method that was 
validated according to the European Medicines Agency bioanalytical method validation guidance (21 July 2011) [19]. A stable isotope label $\left(\left[{ }^{13} \mathrm{C} 2, \mathrm{D} 3\right]\right.$-roxadustat) was used as an internal standard. Roxadustat and the internal standard were extracted from $100 \mu$ l plasma by solid-phase extraction and separated by reversed-phase liquid chromatography on a $\mathrm{C}_{18}$ column using a gradient of water/ acetonitrile; formic acid was used as the mobile phase. Detection was performed via tandem mass spectrometry on a 4000 QTrap mass spectrometer using positive Turbo ion spray ionization. The method was linear in the range of $1-1000 \mathrm{ng} / \mathrm{mL}$; the lower limit of quantification was $1 \mathrm{ng} /$ $\mathrm{mL}$. The method was selective and did not show any evidence of carryover. The inter-run accuracy varied between -4.6 and $10.0 \%$ and the inter-run precision ranged between 0.7 and $4.2 \%$. Concentrations of omeprazole in human Li-heparin plasma were also measured using a liquid chromatography tandem mass spectrometry method. A stable isotope labeled omeprazol-D3 was used as an internal standard. Omeprazole and the internal standard were extracted from $100 \mu \mathrm{l}$ plasma by liquid-liquid extraction and separated by reversed-phase liquid chromatography on a C18 column. Detection was performed via tandem mass spectrometry on an API 4000 mass spectrometer using positive Turbo ion spray ionization. The method was linear in the range of $1-500 \mathrm{ng} / \mathrm{mL}$ with a lower limit of quantification of $1 \mathrm{ng} / \mathrm{mL}$. The method was selective and did not show any evidence of carryover. The inter-run accuracy varied between -1.8 and $4.9 \%$ and the inter-run precision ranged between 2.4 and 9.3\%. Pharmacokinetic parameters were calculated using Phoenix ${ }^{\circledR}$ 6.2.1 (Certara, LP, St. Louis, MO, USA); non-compartmental methods were used to calculate the values of the pharmacokinetic parameters.

\subsubsection{Pharmacokinetic Endpoints}

Primary endpoints were area under the concentration-time profile from the time of dosing extrapolated to infinity $\left(A U C_{i n f}\right)$ and maximum concentration $\left(\mathrm{C}_{\max }\right)$ for roxadustat in plasma. Secondary endpoints included area under the concentration-time profile to the last measurable concentration $\left(\mathrm{AUC}_{\text {last }}\right)$, time to reach maximum concentration $\left(\mathrm{t}_{\max }\right)$, and $\mathrm{t}_{1 / 2}$.

\subsubsection{Safety}

Safety was assessed by evaluation of the nature, frequency, and severity of treatment-emergent adverse events (TEAEs), vital signs (blood pressure and pulse), clinical laboratory tests (hematology, biochemistry, and urinalysis), and routine 12-lead electrocardiogram (ECG). TEAEs were graded according to the National Cancer Institute Common Terminology Criteria for Adverse Event guidelines (version 4.03) and were coded using Medical Dictionary for Regulatory Activities (MedDRA).

\subsection{Statistical Methods}

Based on a previous food-effect study (data on file) that showed a within-subject coefficient of variation of $16 \%$, a sample size of 16 was determined to provide $90 \%$ power (NQUERY 7.0) to demonstrate the absence of effect of omeprazole on roxadustat pharmacokinetics. The absence of effect was to be concluded if the $90 \%$ confidence intervals (CIs) for the ratio of geometric means (i.e., roxadustat + omeprazole/roxadustat alone) for both $\mathrm{AUC}_{\mathrm{inf}}$ and $\mathrm{C}_{\max }$ were within the no-effect boundaries of 80.0 and $125.0 \%$. A total of 18 subjects were enrolled to allow for dropouts.

The safety analysis set (SAF) comprised all subjects who received at least one dose of roxadustat. The pharmacokinetic analysis set, used for all pharmacokinetic analysis, included subjects who received at least one dose of study drug and had plasma concentration data available to determine at least one pharmacokinetic parameter.

Descriptive statistics were used to summarize demographic and baseline data and for pharmacokinetic parameters (i.e., number of subjects, arithmetic mean, standard deviation, $\%$ coefficient of variation, median, and range). Frequencies and percentages were used for categorical data. To assess the impact of omeprazole on the pharmacokinetics of roxadustat, the log-transformed $\mathrm{AUC}_{\mathrm{inf}}$ and $\mathrm{C}_{\max }$ for roxadustat was analyzed using a linear mixed effects model with treatment as a fixed effect and subject as a random effect. No imputation for missing data was performed.

\section{Results}

\subsection{Subject Disposition}

Of 42 subjects screened, 18 were enrolled, and 16 completed the study as per the clinical study protocol. One subject was excluded after receiving one dose of roxadustat during Period 1 due to receiving a prescribed medication (codeine), and one subject withdrew consent after receiving two doses of omeprazole during Period 2. Seventeen subjects $(94.4 \%)$ received omeprazole on Day 1 and 2 of Period 2, and 16 subjects (88.9\%) received omeprazole on Days 3-9 and roxadustat on Day 7 during Period 2. All 18 enrolled subjects were included in the SAF and pharmacokinetic analysis set. Subject baseline characteristics are displayed in Table 1. 


\subsection{Pharmacokinetics of Roxadustat}

The mean plasma concentration-time profiles of roxadustat administered alone (Day 1 of Period 1) and in the presence of omeprazole (Day 7 of Period 2) were similar (Fig. 1). Omeprazole had no significant effect on $\mathrm{AUC}_{\text {last }}$ or $\mathrm{t}_{1 / 2}$ of roxadustat (Table 2). The ratio of geometric means (i.e., roxadustat + omeprazole/roxadustat alone) for both $\mathrm{AUC}_{\mathrm{inf}}$ and $\mathrm{C}_{\max }$ was within the no-effect boundaries of 80.0 and $125.0 \%$, indicating that multiple daily oral doses of $40 \mathrm{mg}$ omeprazole had no significant effect on the pharmacokinetics of roxadustat (Table 2). Pharmacokinetic parameters for omeprazole were obtained to confirm exposure during coadministration with roxadustat. On Day 7, mean trough was $0.494 \mathrm{ng} / \mathrm{mL}$, mean (SD) $\mathrm{C}_{\max }$ was 1207 (705) ng/mL, and mean AUC from the time of dosing to the start of the next dosing interval was 4233 (3615) $\mathrm{ng} \cdot \mathrm{h} / \mathrm{mL}$.

\subsection{Safety}

No serious TEAEs or TEAEs leading to permanent discontinuation of the study drug occurred. A total of six TEAEs were reported in six subjects $(33.3 \%)$ after receiving roxadustat alone, eight TEAEs were reported in

Table 1 Subject baseline characteristics

\begin{tabular}{ll}
\hline Parameter & Total $(n=18)$ \\
\hline Sex, $n(\%)$ & $9(50.0)$ \\
Male & $9(50.0)$ \\
Female & \\
Race, $n(\%)$ & $18(100.0)$ \\
Caucasian & \\
Age (years) & $44.4(9.8)$ \\
Mean (SD) & 48.0 \\
Median & $26-55$ \\
Range & \\
Weight (kg) & $71.1(13.1)$ \\
Mean (SD) & 68.6 \\
Median & $53.8-99.0$ \\
Range & \\
Height (cm) & $170.3(7.4)$ \\
Mean (SD) & 171.5 \\
Median & $159-186$ \\
Range & \\
BMI (kg/m $\left.{ }^{2}\right)$ & $24.3(2.8)$ \\
Mean (SD) & 24.9 \\
Median & $19.3-28.7$ \\
Range &
\end{tabular}

$B M I$ body mass index, $S D$ standard deviation five subjects (29.4\%) after receiving omeprazole alone, and two TEAEs were reported in two subjects $(12.5 \%)$ after receiving roxadustat + omeprazole (Table 3 ). The majority of TEAEs were considered by the investigator to be mild in severity, except for two TEAEs (abdominal pain and headache) reported by two subjects (11.1\%) after receiving roxadustat alone, which were considered moderate in severity. The most commonly reported TEAEs were headache ( $n=3$, roxadustat alone; $n=1$, omeprazole alone), diarrhea ( $n=2$, roxadustat alone; $n=1$, omeprazole alone), and nasopharyngitis ( $n=2$, omeprazole alone; $n=1$, roxadustat + omeprazole). Only one TEAE (dyspepsia/heartburn) reported by one subject after receiving omeprazole alone was considered by the investigator to be possibly related to the study drug, while all other TEAEs were not considered related to the study drug. No clinically significant changes in laboratory results, vital signs, or ECG findings were reported.

\section{Discussion}

The objective of this study was to evaluate the effect of multiple daily oral doses of $40 \mathrm{mg}$ omeprazole on the pharmacokinetics of a single oral dose of $100 \mathrm{mg}$ roxadustat, and to assess the safety and tolerability of a single oral dose of $100 \mathrm{mg}$ roxadustat alone or in combination with multiple daily oral doses of $40 \mathrm{mg}$ omeprazole. The solubility of roxadustat increases with increasing $\mathrm{pH}$ in simulated gastric fluid buffers. Over the $\mathrm{pH}$ range of 1.2-7.5, the in vitro solubility of roxadustat increases from 0.001 to $3.7 \mathrm{mg} / \mathrm{mL}$, suggesting that its absorption may be augmented by the concomitant administration of omeprazole, a commonly used drug that increases gastric $\mathrm{pH}$. However, omeprazole $40 \mathrm{mg}$ administered once daily in healthy subjects has been shown to increase gastric $\mathrm{pH}$ to $\sim 5[20,21]$, and at this $\mathrm{pH}$ the in vitro solubility of roxadustat is still low $(0.006 \mathrm{mg} / \mathrm{mL})$. Therefore, only a fraction of the roxadustat dose would be dissolved in the stomach and a relative change in the absorption of roxadustat would not be detected. The results of this clinical study demonstrate that multiple daily oral doses of $40 \mathrm{mg}$ omeprazole, which is the maximum of the commonly used therapeutic dose range (20-40 $\mathrm{mg}$ once daily), had no significant effect on the pharmacokinetics of a single oral dose of $100 \mathrm{mg}$ roxadustat. The geometric least squares mean ratios for $\mathrm{AUC}_{\mathrm{inf}}$ and $\mathrm{C}_{\max }$ of roxadustat (with omeprazole/alone) were $104.53 \%$ (90\% CI $97.74 \%$, $111.80 \%$ ) and $104.46 \%$ (90\% CI 90.99\%, 119.93\%), respectively, and the $90 \%$ CIs were within the no-effect boundaries of 80.0 and $125.0 \%$. Omeprazole is also an inhibitor of CYP2C19 [18] and is typically associated with CYP-based interactions. Roxadustat is primarily 
Fig. 1 Mean plasma concentration profiles of roxadustat in subjects treated with roxadustat alone $(n=18)$, and roxadustat in the presence of omeprazole $(n=16)$. Error bars indicate standard deviation

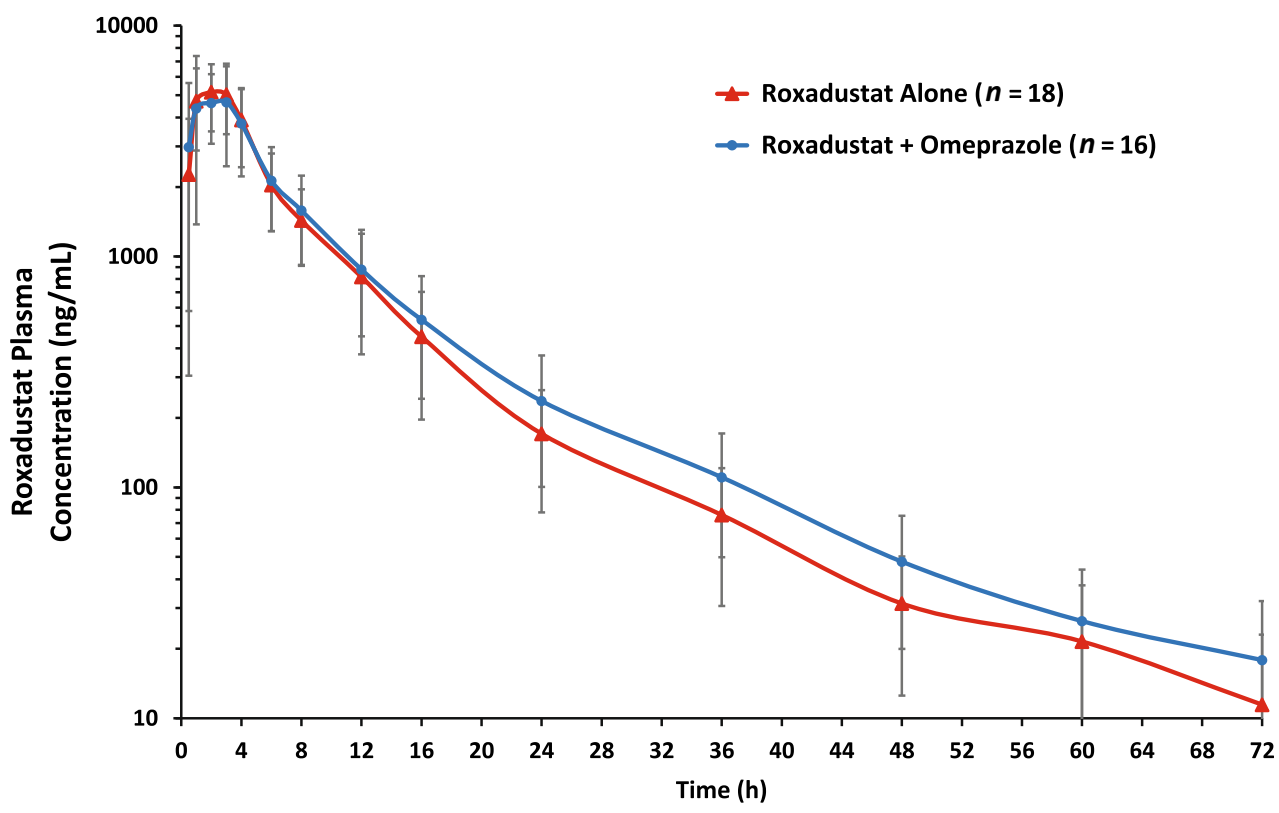

Table 2 Pharmacokinetics of roxadustat

\begin{tabular}{|c|c|c|c|c|c|}
\hline Parameter & $\mathrm{AUC}_{\mathrm{inf}}(\mathrm{ng} \cdot \mathrm{h} / \mathrm{mL})$ & $\mathrm{C}_{\max }(\mathrm{ng} / \mathrm{mL})$ & $t_{\max }(h)$ & $\mathrm{AUC}_{\text {last }}(\mathrm{ng} \cdot \mathrm{h} / \mathrm{mL})$ & $\mathrm{t}_{1 / 2}(\mathrm{~h})$ \\
\hline \multicolumn{6}{|c|}{ Roxadustat alone $(n=18)$} \\
\hline Mean & 37,781 & 6345 & 1.897 & 37,514 & 12.92 \\
\hline $\mathrm{SD}(\% \mathrm{CV})$ & $10,628(28.1)$ & $1298(20.5)$ & 0.7552 (NA) & $10,496(28.0)$ & $4.737(36.7)$ \\
\hline Range & $19,572-57,469$ & $3173-8473$ & $1.00-3.00$ & $19,359-57,265$ & $7.02-22.1$ \\
\hline Median & 35,119 & 6526 & 2.000 & 35,024 & 11.71 \\
\hline \multicolumn{6}{|c|}{ Roxadustat + omeprazole $(n=16)$} \\
\hline Mean & 39,642 & 6716 & 1.758 & 39,276 & 13.23 \\
\hline $\mathrm{SD}(\% \mathrm{CV})$ & $13,317(33.6)$ & $1980(29.5)$ & 1.053 (NA) & $13,205(33.6)$ & $5.18(39.2)$ \\
\hline Range & $23,773-68,729$ & $3632-10,501$ & $0.500-4.02$ & $23,245-67,801$ & $7.42-27.0$ \\
\hline Median & 37,688 & 6661 & 1.500 & 38,262 & 12.02 \\
\hline \multicolumn{6}{|c|}{ Geometric least squares mean ratio (roxadustat + omeprazole/roxadustat alone) } \\
\hline$\%$ & 104.53 & 104.46 & & & \\
\hline $90 \%$ CI & $97.74-111.80$ & 90.99-119.93 & & & \\
\hline
\end{tabular}

All means presented are arithmetic

$A U C_{i n f}$ area under the concentration-time profile from the time of dosing extrapolated to infinity, $A U C_{\text {last }}$ area under the concentration-time profile to the last measurable concentration, $C I$ confidence interval, $C_{\max }$ maximum concentration, $C V$ coefficient of variation, $N A$ not applicable, $N C$ not calculated, $S D$ standard deviation, $t_{1 / 2}$ terminal elimination half-life, $t_{\max }$ time to reach maximum concentration

metabolized via phase I oxidation and phase II conjugation and is not a substrate for CYP2C19; therefore, no CYPmediated drug interactions were anticipated in this study. The combination of multiple daily oral doses of omeprazole with roxadustat was considered safe, and the majority of TEAEs reported were mild in severity. No serious TEAEs or TEAEs leading to permanent discontinuation of study drug or clinically significant changes in laboratory results, vital signs, or ECG findings occurred.

The lack of a significant effect of omeprazole on the pharmacokinetics of roxadustat indicates that an increase of gastric $\mathrm{pH}$ has no significant effect on the absorption of roxadustat, and suggests that the pharmacokinetics of roxadustat may not be impacted by other gastric $\mathrm{pH}$-raising agents.

\section{Conclusions}

In healthy subjects, multiple daily oral doses of $40 \mathrm{mg}$ omeprazole had no significant effect on the pharmacokinetics of a single oral dose of $100 \mathrm{mg}$ roxadustat. 
Table 3 Treatment-emergent adverse events

\begin{tabular}{llll}
\hline $\begin{array}{l}\text { MedDRA v15.1 system organ class } \\
\text { Preferred term }\end{array}$ & $\begin{array}{l}\text { Roxadustat alone } \\
(n=18)\end{array}$ & $\begin{array}{l}\text { Omeprazole alone } \\
(n=17)\end{array}$ & $\begin{array}{l}\text { Roxadustat }+ \text { omeprazole } \\
(n=16)\end{array}$ \\
\hline $\begin{array}{l}\text { Number of subjects with TEAE }{ }^{\mathrm{a}} \\
\text { Gastrointestinal disorders }\end{array}$ & $6(33.3)$ & $5(29.4)$ & $2(12.5)$ \\
Abdominal pain & $3(16.7)$ & $3(17.6)$ & 0 \\
Constipation & $1(5.6)$ & 0 & 0 \\
Diarrhea & 0 & $1(5.9)$ & 0 \\
Dyspepsia & $2(11.1)$ & $1(5.9)$ & 0 \\
Infections and infestations & 0 & $1(5.9)^{\mathrm{b}}$ & 0 \\
Nasopharyngitis & 0 & $3(17.6)$ & $1(6.3)$ \\
Oral herpes & 0 & $2(11.8)$ & $1(6.3)$ \\
Investigations & 0 & $1(5.9)$ & 0 \\
Lymph node palpable & 0 & 0 & $1(6.3)$ \\
Nervous system disorders & 0 & 0 & $1(6.3)$ \\
Headache & $3(16.7)$ & $1(5.9)$ & 0 \\
Respiratory, thoracic, and mediastinal disorders & $3(16.7)$ & $1(5.9)$ & 0 \\
Oropharyngeal pain & 0 & $1(5.9)$ & 0 \\
\hline
\end{tabular}

Data are presented as $n(\%)$

MedDRA Medical Dictionary for Regulatory Activities, TEAE treatment-emergent adverse event

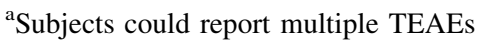

${ }^{\mathrm{b}}$ Considered to be possibly related to study drug

Roxadustat was considered safe and well tolerated when administered alone or in combination with multiple daily oral doses of $40 \mathrm{mg}$ omeprazole.

Acknowledgements Medical writing and editorial assistance, under the guidance of the authors, was provided by SuccinctChoice Medical Communications (Chicago, IL) and funded by Astellas Pharma, Inc. Roxadustat is being developed by FibroGen, AstraZeneca, and Astellas. The authors would like to thank Jan Noukens, from Venn Life Sciences (Breda, The Netherlands), for his support with the pharmacokinetic analysis and reporting for this study.

\section{Compliance with Ethical Standards}

Funding This study was sponsored and monitored by Astellas Pharma, Inc.

Conflict of interest Dorien Groenendaal-van de Meent, Martin den Adel, Jan van Dijk, Begona Barroso-Fernandez, Rachid El Galta, and Marloes Schaddelee are employees of Astellas Pharma Europe B.V.; Georg Golor was the principal investigator for this study and was employed with PAREXEL Berlin at the time the study was conducted.

Ethical approval All procedures performed in studies involving human participants were in accordance with the ethical standards of the institutional and/or national research committee and with the 1964 Helsinki declaration and its later amendments or comparable ethical standards. The protocol was approved by an Independent Ethics Committee (Landesamt für Gesundheit und Sociales [Ethik-Kommission des Landes Berlin].
Informed consent Informed consent was obtained from all individual participants in the study.

Open Access This article is distributed under the terms of the Creative Commons Attribution-NonCommercial 4.0 International License (http://creativecommons.org/licenses/by-nc/4.0/), which permits any noncommercial use, distribution, and reproduction in any medium, provided you give appropriate credit to the original author(s) and the source, provide a link to the Creative Commons license, and indicate if changes were made.

\section{References}

1. Babitt JL, Lin HY. Mechanisms of anemia in CKD. J Am Soc Nephrol. 2012;23(10):1631-4.

2. Li Y, Shi H, Wang WM, Peng A, Jiang GR, Zhang JY, et al. Prevalence, awareness, and treatment of anemia in Chinese patients with nondialysis chronic kidney disease: first multicenter, cross-sectional study. Medicine (Baltim). 2016;95(24):e3872.

3. Stauffer ME, Fan T. Prevalence of anemia in chronic kidney disease in the United States. PLoS One. 2014;9(1):e84943.

4. Covic A, Jackson J, Hadfield A, Pike J, Siriopol D. Real-world impact of cardiovascular disease and anemia on quality of life and productivity in patients with non-dialysis-dependent chronic kidney disease. Adv Ther. 2017;34(7):1662-72.

5. Garlo K, Williams D, Lucas L, Wong R, Botler J, Abramson S, et al. Severity of anemia predicts hospital length of stay but not readmission in patients with chronic kidney disease: a retrospective cohort study. Medicine (Baltim). 2015;94(25):e964. 
6. Eriksson D, Goldsmith D, Teitsson S, Jackson J, van Nooten F. Cross-sectional survey in CKD patients across Europe describing the association between quality of life and anaemia. BMC Nephrol. 2016;17(1):97.

7. Gupta N, Wish JB. Hypoxia-inducible factor prolyl hydroxylase inhibitors: a potential new treatment for anemia in patients with CKD. Am J Kidney Dis. 2017;69(6):815-26.

8. Del Vecchio L, Locatelli F. An overview on safety issues related to erythropoiesis-stimulating agents for the treatment of anaemia in patients with chronic kidney disease. Expert Opin Drug Saf. 2016;15(8):1021-30.

9. Besarab A, Provenzano R, Hertel J, Zabaneh R, Klaus SJ, Lee T, et al. Randomized placebo-controlled dose-ranging and pharmacodynamics study of roxadustat (FG-4592) to treat anemia in nondialysis-dependent chronic kidney disease (NDD-CKD) patients. Nephrol Dial Transplant. 2015;30(10):1665-73.

10. Locatelli F, Fishbane S, Block GA, Macdougall IC. Targeting hypoxia-inducible factors for the treatment of anemia in chronic kidney disease patients. Am J Nephrol. 2017;45(3):187-99.

11. Besarab A, Chernyavskaya E, Motylev I, Shutov E, Kumbar LM, Gurevich K, et al. Roxadustat (FG-4592): correction of Anemia in Incident Dialysis Patients. J Am Soc Nephrol. 2016;27(4):1225-33.

12. Chen N, Qian J, Chen J, Yu X, Mei C, Hao C, et al. Phase 2 studies of oral hypoxia-inducible factor prolyl hydroxylase inhibitor FG-4592 for treatment of anemia in China. Nephrol Dial Transplant. 2017;32(8):1373-86.

13. Provenzano R, Besarab A, Wright S, Dua S, Zeig S, Nguyen P, et al. Roxadustat (FG-4592) versus epoetin alfa for anemia in patients receiving maintenance hemodialysis: a phase 2 , randomized, 6- to 19-week, open-label, active-comparator, doseranging, safety and exploratory efficacy study. Am J Kidney Dis. 2016;67(6):912-24.

14. Provenzano R, Besarab A, Sun CH, Diamond SA, Durham JH, Cangiano JL, et al. Oral hypoxia-inducible factor prolyl hydroxylase inhibitor roxadustat (FG-4592) for the treatment of anemia in patients with CKD. Clin J Am Soc Nephrol. 2016;11(6):982-91.

15. Groenendaal-van de Meent D, Adel MD, Noukens J, Rijnders S, Krebs-Brown A, Mateva L, et al. Effect of moderate hepatic impairment on the pharmacokinetics and pharmacodynamics of roxadustat, an oral hypoxia-inducible factor prolyl hydroxylase inhibitor. Clin Drug Investig. 2016;36(9):743-51.

16. Groenendaal-van de Meent D, den Adel M, Rijnders S, KrebsBrown A, Kerbusch V, Golor G, et al. The hypoxia-inducible factor prolyl-hydroxylase inhibitor roxadustat (FG-4592) and warfarin in healthy volunteers: a pharmacokinetic and pharmacodynamic drug-drug interaction study. Clin Ther. 2016;38(4):918-28.

17. Antra MUPS ${ }^{\circledR}$ [package insert]. Wedel, Germany: AstraZeneca; 2013. https://www.medicines.org.uk/emc/files/pil.1493.pdf. Accessed 26 June 2017.

18. PRILOSEC ${ }^{\circledR}$ (omeprazole) [package insert]. Wilmington, DE: AstraZeneca; 2016. https://www.azpicentral.com/prilosec/prilosec. pdf\#page $=1$. Accessed 26 June 2017.

19. European Medicines Agency. Guideline on bioanalytical method validation. Committee for Medicinal Products for Human Use (CHMP). 2011.http://www.ema.europa.eu/docs/en_GB/document_ library/Scientific_guideline/2011/08/WC500109686.pdf. Accessed 26 June 2017.

20. Gan KH, Geus WP, Lamers CB, Heijerman HG. Effect of omeprazole $40 \mathrm{mg}$ once daily on intraduodenal and intragastric $\mathrm{pH}$ in $\mathrm{H}$. pylori-negative healthy subjects. Dig Dis Sci. 1997;42(11):2304-9.

21. Prichard PJ, Yeomans ND, Mihaly GW, Jones DB, Buckle PJ, Smallwood RA, et al. Omeprazole: a study of its inhibition of gastric $\mathrm{pH}$ and oral pharmacokinetics after morning or evening dosage. Gastroenterology. 1985;88(1 Pt 1):64-9. 